\title{
Ultrathin Carbon Nanotube With Single, Double, and Triple Bonds
}

\author{
E. Menéndez-Proupin, ${ }^{1,2}$ Ana L. Montero-Alejo, ${ }^{1,3}$ and J. M. García de la Vega ${ }^{1}$ \\ ${ }^{1}$ Departamento de Química Física Aplicada, Facultad de Ciencias, Universidad Autónoma de Madrid, 28049 Madrid, Spain \\ ${ }^{2}$ Departamento de Física, Facultad de Ciencias, Universidad de Chile, Las Palmeras 3425, 780-0024 Ñuñoa, Santiago, Chile \\ ${ }^{3}$ Laboratorio de Química Computacional y Teórica, Facultad de Química, Universidad de la Habana, 10400 Havana, Cuba
}

(Received 1 June 2012; published 4 September 2012)

\begin{abstract}
A metastable carbon nanotube with single, double, and triple bonds has been predicted from $a b$ initio simulation. It results from the relaxation of an ideal carbon nanotube with chirality $(2,1)$, without any potential barrier between the ideal nanotube and the new structure. Ten-membered carbon rings are formed by breaking carbon bonds between adjacent hexagons; eight-membered rings, already present in the ideal structure, become the smallest rings. This structure is stable in molecular dynamics simulations at temperatures up to $1000 \mathrm{~K}$. Raman, infrared, and optical absorption spectra are simulated to allow its identification in the laboratory. The structure can be described as a double helical chain with alternating single, double, and triple bonds, where the chains are bridged by single bonds.
\end{abstract}

DOI: 10.1103/PhysRevLett.109.105501

Ultrathin carbon nanotubes (CNT) with diameter smaller than $3 \AA$ are generally believed to be unstable due to the large distortion of their covalent bonds from the $s p_{2}$ trigonal geometry. However, the immense variety of carbon nanostructures are determined by kinetics rather than thermodynamics, and the properties of ultrathin CNT's have been explored in the recent literature [1]. A related kind of objects is linear carbon chains (LCC), which have generated great interest because of their possible applications in carbon nanoelectronics [2-4], and also for their observation in the interstellar medium $[5,6]$. LCC present coexisting $s p$ and $s p^{2}$ hybridization and offer plausible ways to tailor their properties. We have found a metastable structure that deviates from the folded graphene model showing 10-atom rings and several bi-coordinated carbon atoms. This structure can be regarded as a double helical chain with alternating single, double, and triple bonds. In this Letter, we present a description of this structure and its electronic properties. As the carbon nanostructures are generally identified by means of spectroscopy, we also present calculations of the optical absorption, infrared (IR) absorption, and Raman spectra. These calculations have been carried out with the QUANTUM ESPRESSO [7] package in the framework of density functional theory (DFT) [8].

The chiral CNT $(2,1)$ may have its ideal folded-graphene structure at the cost of having some bond angles as small as $97^{\circ}$ and some torsion angles as large as $67^{\circ}$. Our carbon structure was obtained through a variable cell relaxation of the ideal CNT $(2,1)$. The obtained structure resulted from breaking bonds that join adjacent hexagons, to form 10-atoms rings, as shown in Fig. 1. Eight-membered rings inherited from the ideal $(2,1)$ structure become the smallest and closest path connecting carbons. We will name this structure after the highest-membered ring, CNT10R. CNT10R still has a tubular shape, with an outer diameter
PACS numbers: 61.48.De, 31.15.A-, 63.22.Gh, 78.67.Ch

of $3.2 \AA$ (measured by the nuclear positions). CNT10R is $21 \mathrm{eV}$ below CNT $(2,1)$ in total energy per unit cell (28 atoms). There is no energy barrier between both structures in our DFT calculations. If the energy is calculated with the Brenner classical bond-order interatomic potential [9], there is an energy barrier, and the straightforward relaxation preserves the CNT at a local energy minimum with $(2,1)$ geometry. However, the CNT10R structure has lower energy, $14 \mathrm{eV}$ less than the starting CNT $(2,1)$ structure. Note that CNT $(2,1)$ has been reported to be stable when optimized with the semiempirical parametric method 3 (PM3) [1], and that semiempirical methods are less accurate than DFT for classes of molecules not included in parametrization.

The carbon bond distances display features of single, double, and triple bonds. Let us indicate the coordination of a carbon by a superscript $n$ in the notation ${ }^{n} \mathrm{C}$. We have shown the bond distances in Table I and Fig. 1. The ${ }^{3} \mathrm{C}$ (trifold coordinated) atoms form a single chain that has some importance on the Raman spectrum, as discussed

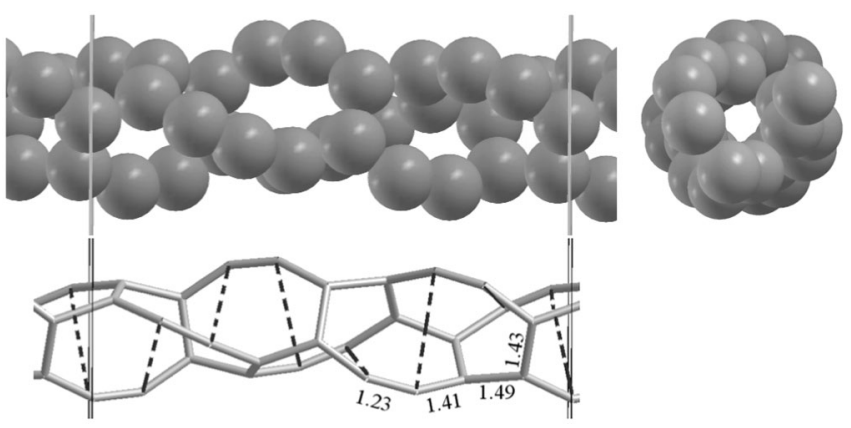

FIG. 1. Top: Structure of the CNT10R nanotube in front and lateral view. The vertical lines indicate the unit cell. Bottom: Stick view with the broken bonds are indicated by dashed lines (2.83 $\AA$ in length). The bond distances are indicated in $\AA$ units. 
below. There are two types of ${ }^{3} \mathrm{C}-{ }^{3} \mathrm{C}$ bonds. The shortest one is oriented almost transversely to the nanotube axis, while the longest one is almost longitudinal.

We have tested the stability of CNT10R in two ways. First, we performed ab initio relaxation from CNT $(2,1)$ with a triple unit cell (72 atoms) and obtained the same structure. Second, we performed a series of classical molecular dynamics runs [8] at different temperatures, and found that the CNT10R structure is stable at $1000 \mathrm{~K}$. We used supercells containing up to 140 atoms (five unit cells) and time intervals up to $1 \mathrm{~ns}$. As a cross-check, we relaxed the last configuration obtained from MD using DFT, and obtained the same structure and the same energy per unit cell (within $1 \mathrm{mRy}$ ) as for the single and triple unit cells. We also tested the effect of dispersion forces using DFT-D correction [10]. Dispersion forces have a negligible effect, decreasing the energy by only $0.054 \mathrm{eV} /$ atom and the periodicity length by $0.3 \%$.

CNT10R is a closed-shell system in its ground state. Figure 2 shows the DFT band diagram and the DOS in the region around the fundamental gap. The lowest gap is $1.23 \mathrm{eV}$, indirect between the valence band that is maximum at the $\Gamma$ point and the conduction band minimum at $0.85 \pi / a$ ( $a$ is the lattice constant). The direct gap at the $\Gamma$ point is $1.35 \mathrm{eV}$. DOS shows the van Hove singularities that are common in one-dimensional systems. From the projected DOS [8], we find that between $-11 \mathrm{eV}$ and $3 \mathrm{eV}$, DOS is dominated by $2 p$ states. Out of this range, $2 s$ states contribute significantly and dominate DOS peaks at -18.3 , -14.8 , and $-14.6 \mathrm{eV}$. The projected DOS on tri- and bi-coordinated carbons is also shown in Ref. [8]. The largest imbalance is in favor of bi-coordinated carbons at $-2.35 \mathrm{eV}$ and tri-coordinated carbons at $-7.13 \mathrm{eV}$.

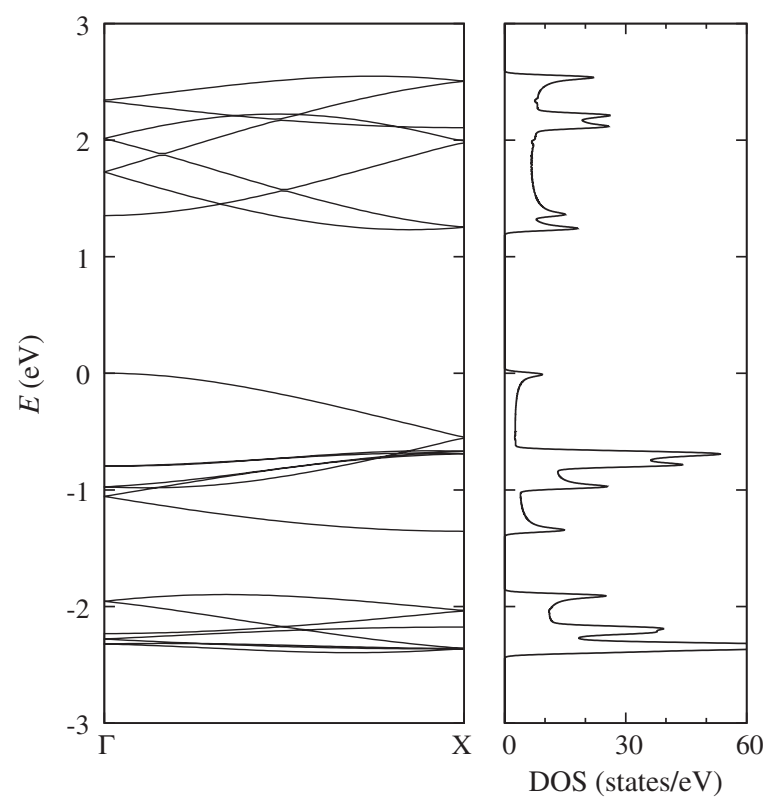

FIG. 2. Band diagram and density of states of CNT10R structure. Zero energy is the Fermi level.
This imbalance might help us to differentiate both types of carbons using scanning tunneling microscopy. Globally, there is practically no charge transfer between tri- and bi-coordinated carbons, as indicated by the Löwdin charges. A ionization potential of $5.71 \mathrm{eV}$ has been estimated as the difference between the electrostatic potential far from the nanotube and the highest occupied state. DFT values for ionization potentials are systematically low [11], and a correct value should be about $7.5 \mathrm{eV}$. Table I shows the bond orders computed with a localized basis set [8]. These bond orders match the bond multiplicities expected from the bond lengths. Further analysis of charge density, spin density, and the electron localization function reveals the absence of dangling bonds and lone pairs [8].

The optical absorption spectrum calculated using the time-dependent DFT (TDDFT) approach is presented in Fig. 3. As usual with one-dimensional objects, there is pronounced anisotropy, and the absorption is much stronger for the light polarization parallel to the tube axis. As the optical gap is larger for perpendicular polarization, the perpendicular polarization spectrum should be undetectable in practical cases. It is tempting to ascribe the absorption peaks to the transitions between the van-Hove singularities of the single-particle DOS shown in Fig. 2. However, the sampling of equivalent $\mathrm{k}$ points obtained with our seven unit-cell model cannot reproduce the van Hove structure of the DOS, which was computed with $60 \mathrm{k}$ points. We believe that our calculations select the main features of the absorption spectrum. This is supported by our additional calculations using 3-, 5-, and 8-unit supercells, as well as the calculations in the independentelectron approximation [12] performed using the EPSILON post-processing code of QUANTUM ESPRESSO that allows $k$-point sampling. With this approach, we used up to $28 \mathrm{k}$ points to sample the Brillouin zone and found that the same polarizability can be obtained with $10 k$-point sampling [13]. With seven $k$ points, there are some small differences. The doublet structure at 1.37 and $1.48 \mathrm{eV}$ appears as a single peak with 10 or more $k$ points, and the peak at $2.31 \mathrm{eV}$ is reduced to a small shoulder. In fact, independent electron transitions produce an absorption spectrum with the same structure that the TDDFT approach produces. The main differences are that the TDDFT approach decreases the intensity of the bands at 1.4 and $1.98 \mathrm{eV}$, and the increment of the band at $3.59 \mathrm{eV}$. Moreover, the absorption for perpendicular polarization is strongly reduced in TDDFT.

TABLE I. Carbon bond lengths (BL) and bond orders (BO) in CNT10R. ${ }^{n} \mathrm{C}$ indicates an $n$-coordinated carbon.

\begin{tabular}{lccccc}
\hline \hline & ${ }^{2} \mathrm{C}-{ }^{2} \mathrm{C}$ & ${ }^{2} \mathrm{C}-{ }^{3} \mathrm{C}$ & ${ }^{3} \mathrm{C}-{ }^{3} \mathrm{C}$ & ${ }^{3} \mathrm{C}-{ }^{3} \mathrm{C}$ & Graphene \\
\hline BL $(\AA)$ & 1.23 & 1.41 & 1.43 & 1.49 & 1.42 \\
BO & 2.46 & 1.21 & 1.45 & 1.00 & 1.24 \\
\hline \hline
\end{tabular}




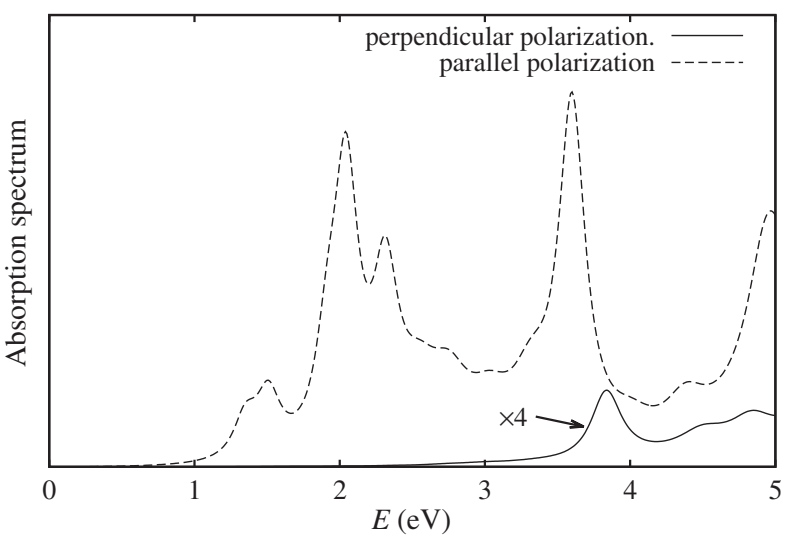

FIG. 3. Absorption spectrum of CNT10R structure.

Bearing this in mind, we can state that the peak at $1.37 \mathrm{eV}$ matches the $1.35 \mathrm{eV}$ direct bandgap at the $\Gamma$ point. Moreover, the peak at $1.48 \mathrm{eV}$ matches the vertical transitions between the top valence and the bottom conduction bands along the $\Gamma-X$ direction. The peak at $1.98 \mathrm{eV}$ is related to the transitions between the van Hove singularities at $-0.7 \mathrm{eV}$ and $1.23 \mathrm{eV}$, i.e., vertical transitions from the bunch of valence bands at $k \sim 0.85 \pi / a, E \sim-0.7 \mathrm{eV}$ to the conduction band minimum. The peak at $3.59 \mathrm{eV}$ seems to be related to the transitions between the van Hove singularities at -2.19 and $1.35 \mathrm{eV}$ or from -2.34 to $1.23 \mathrm{eV}$. In the first explanation, the transition would occur at $k \sim 0$, starting from the band at $-2.23 \mathrm{eV}$, which has the same curvature as the conduction band. In the second alternative, the transition would be at $k \sim 0.85 \pi / a$, from the lowest valence bands of Fig. 2 to the conduction band minimum.

Nonresonant Raman intensities are shown in Fig. 4. Raman tensors are predominantly diagonal [14], and the $z z$-component (along the tube axis) is one or more orders of magnitude larger than the perpendicular components. Therefore, Raman intensities are maximal for the incoming and scattered light polarized parallel to the tube axis. The pattern is not expected to change for nonparallel polarizations, and the overall intensity should be proportional to $\cos ^{2} \theta_{i} \cos ^{2} \theta_{s}$, where $\theta_{i}$ and $\theta_{s}$ are the angles between

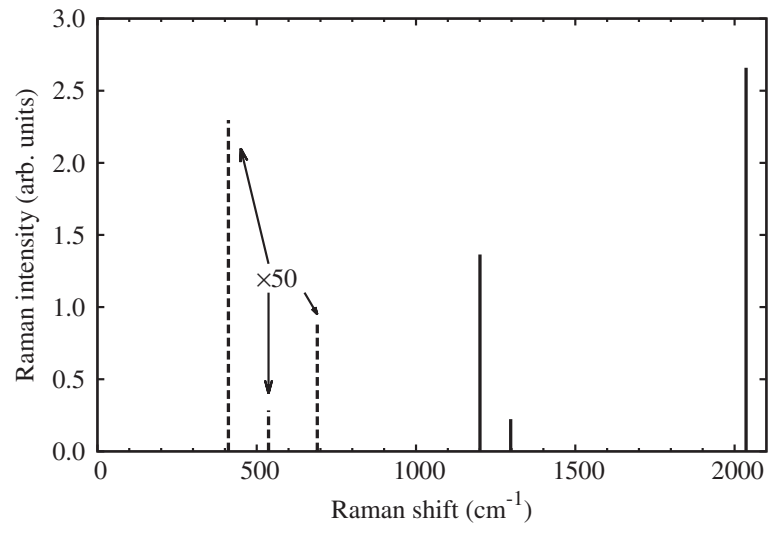

FIG. 4. Nonresonant Raman spectrum of CNT10R. the incoming and the scattered light polarization vectors, respectively. A similar orientational dependence has been demonstrated for resonance Raman scattering [15]. The strongest Raman lines, in order of decreasing intensity, are at 2036, 1201, and $1297 \mathrm{~cm}^{-1}$. The amplitudes of Raman and IR active modes can be seen in the Supplemental Material [8]. The mode at $2036 \mathrm{~cm}^{-1}$ is principally due to stretching all the ${ }^{2} \mathrm{C}-{ }^{2} \mathrm{C}$ bonds, and this represents a spectroscopical signature of the triple bond. The mode at $1201 \mathrm{~cm}^{-1}$ is due to stretching of the transversal ${ }^{3} \mathrm{C}-{ }^{3} \mathrm{C}$ bonds. Alternatively, this mode is described as transversal vibrations of the ${ }^{3} \mathrm{C}$ chain. The mode at $1297 \mathrm{~cm}^{-1}$ is produced by the longitudinal vibrations of the ${ }^{3} \mathrm{C}$ chain, with every ${ }^{3} \mathrm{C}$ vibrating in antiphase with its closest neighbors. The frequency of $1297 \mathrm{~cm}^{-1}$ coincides with the so-called $D$ line observed in nanotubes and graphite, which is generally attributed to defects $[16,17]$. There are three more modes, at 411, 537, and $690 \mathrm{~cm}^{-1}$, with Raman intensities $1 / 5,1 / 40$, and $1 / 13$, respectively, of the $1297 \mathrm{~cm}^{-1}$ line. These modes are shown in Fig. 4 with their intensities multiplied by 50 . We did not find a radial breathing mode in this structure, and hence there is no corresponding Raman line. Moreover, there are no phonon modes between 1409 and $2036 \mathrm{~cm}^{-1}$, hence the ubiquitous $G$ band around $1582 \mathrm{~cm}^{-1}$ is absent in CNT10R. The absence of these modes is a manifestation of the lack of purely $s p^{2}$ carbons in CNT10R.

The IR spectrum presents lines at $279,488,711$, and $1293 \mathrm{~cm}^{-1}$, in all cases for polarization parallel to the tube axis. Figure 5 shows the IR activity $f^{\mathrm{IR}}=$ $\left|\partial \mathbf{d} / \partial q_{\nu}\right|^{2}$, where $\mathbf{d}$ is the dipole moment induced by the normal mode with coordinate $q_{\nu}$ [18]. For perpendicular polarization, the IR intensities are about 50 times smaller, and are not shown. IR signals are generally weak on carbon nanotubes owing to the absence of a static charge. Hence, the IR absorption for perpendicular polarization is probably unobservable in experiments. This pattern of IR spectrum is quite different from predictions and observations in carbon nanotubes, graphene, and graphene nanoribbons [19-22]. The mode at $711 \mathrm{~cm}^{-1}$ is the strongest one, similar to the $D$ band in nanotubes,

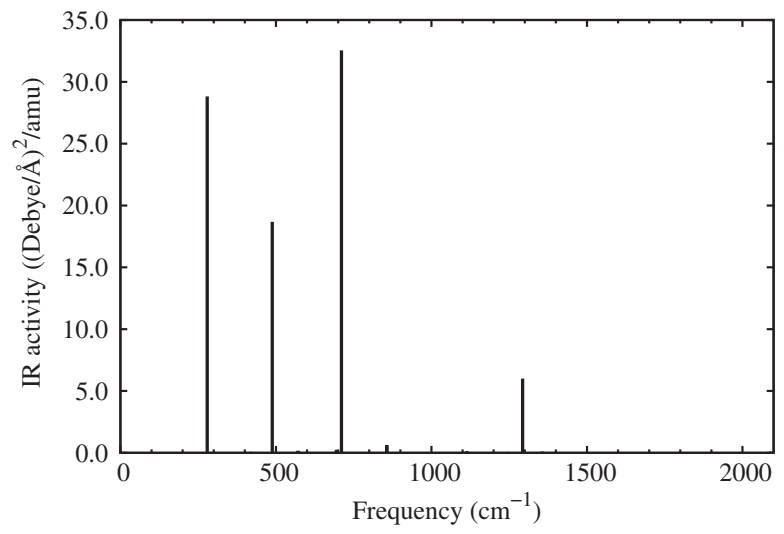

FIG. 5. IR activity of a CNT10R unit. 
but it can be distinguished since its frequency is about $150 \mathrm{~cm}^{-1}$ lower. Moreover, the low-frequency modes at 279 and $488 \mathrm{~cm}^{-1}$ have large intensities without equivalence in the related systems mentioned. The order of magnitude of IR activity is the same [23] as that calculated in Ref. [22] for graphene nanoribbons. We also computed the IR activity of an $(8,0)$ CNT, which has been reported to display anomalously big IR intensities and Born effective charge tensors [21], and found the same order of magnitudes as for CNT10R.

The electronic and vibrational properties of CNT10R resemble those of linear carbon chains with coexisting $s p$ and $s p^{2}$ hybridization $[3,4]$. CNT10R can be regarded as a pair of twinned chains bridged by single bonds between tri-coordinated carbons, as shown in Fig. 6 of Ref. [8]. Each chain has a periodicity of twice the unit cell, and is linked through single bonds between tri-coordinated carbons. The bond multiplicities are idealized in this picture; the bond angles along the chains are not $180^{\circ}$ and $120^{\circ}$, and some amount of bond order is transferred from the triple to the adjacent single bonds.

While CNT10R may be identified in a lucky experiment, a possible route for the synthesis of CNT10R may be the use of reactions inside a protecting single-walled CNT. For example, annealing ferrocene peapods allow to obtain a variety of ultrathin and mobile CNT with the minimal theoretical (observed) diameters of 2.5 (3.9) $\AA$ [24].

In summary, we have predicted the existence and optical properties of a carbon tubular nanostructure that is stable for temperatures up to $1000 \mathrm{~K}$. The structure can be regarded as an ultrathin carbon nanotube of chirality $(2,1)$ with broken bonds. Due to the broken bonds, the structure presents 10-atom rings with single, double, and triple bonds. Triple bonds link bi-coordinated carbons that always appear in dimers. The tri-coordinated carbons form a unique helical chain with periodicity length of $11.15 \AA$. The optical, IR, and Raman spectra present a strong anisotropy that favors configurations with light electric polarization vector parallel to the tube axis. The pattern of Raman and IR spectra differs significantly from the spectra of other carbon structures like graphene, nanoribbons, and standard nanotubes.

This work was supported by Grants No. SB2010-0119 (MEC), No. CTQ2010-19232 (MICIN), and No. A1/ 035856/11 (AECID). P. Giannozzi， R. Gebauer, D. Rocca, and S. Binnie are acknowledged for assistance with the theory and the codes. B. Hernández, R. Wannemacher, and J. Gierschner are acknowledged for a fruitful discussion. The Center of Bioinformatics and Molecular Simulation at the University of Talca is acknowledged for computer time, as well as the technical assistance of D. Aguayo and L. Monsalve.
[1] M.-F. Ng, S. L. Sun, and R. Q. Zhang, Phys. Rev. B 72, 033406 (2005).

[2] C. Jin, H. Lan, L. Peng, K. Suenaga, and S. Iijima, Phys. Rev. Lett. 102, 205501 (2009).

[3] L. Ravagnan, N. Manini, E. Cinquanta, G. Onida, D. Sangalli, C. Motta, M. Devetta, A. Bordoni, P. Piseri, and P. Milani, Phys. Rev. Lett. 102, 245502 (2009).

[4] G. Moras, L. Pastewka, M. Walter, J. Schnagl, P. Gumbsch, and M. Moseler, J. Phys. Chem. C 115, 24653 (2011).

[5] M. Tulej, D. A. Kirkwood, M. Pachkov, and J. P. Maier, Astrophys. J. 506, L69 (1998).

[6] M. Larsson, W. D. Geppert, and G. Nyman, Rep. Prog. Phys. 75, 066901 (2012).

[7] P. Giannozzi et al., J. Phys. Condens. Matter 21, 395502 (2009).

[8] See Supplemental Material at http://link.aps.org/ supplemental/10.1103/PhysRevLett.109.105501 for details on the calculations and additional information on geometry, electronic structure, and vibrational modes.

[9] D. Brenner, O. Shenderova, J. Harrison, S. Stuart, B. Ni, and S. Sinnott, J. Phys. Condens. Matter 14, 783 (2002).

[10] S. Grimme, J. Comput. Chem. 27, 1787 (2006).V. Barone, M. Casarin, D. Forrer, M. Pavone, M. Sambi, and A. Vittadini, J. Comput. Chem. 30, 934 (2009).

[11] X. Blase, C. Attaccalite, and V. Olevano, Phys. Rev. B 83, 115103 (2011).

[12] R. Colle, P. Parruccini, A. Benassi, and C. Cavazzoni, J. Phys. Chem. B 111, 2800 (2007).

[13] Computer resources limited our TDDFT calculation to a maximal eight-unit supercell, using 80 processor cores during four days and up to $160 \mathrm{~GB}$ of random access memory.

[14] Due to lack of symmetry, the nondiagonal components are not exactly zero, but they are several orders of magnitudes smaller than the diagonal components.

[15] J. Hwang, H. H. Gommans, A. Ugawa, H. Tashiro, R. Haggenmueller, K. I. Winey, J. E. Fischer, D. B. Tanner, and A. G. Rinzler, Phys. Rev. B 62, R13310 (2000).

[16] G. S. Duesberg, I. Loa, M. Burghard, K. Syassen, and S. Roth, Phys. Rev. Lett. 85, 5436 (2000).

[17] M. Hulman, R. Pfeiffer, and H. Kuzmany, New J. Phys. 6, 1 (2004).

[18] D. Porezag and M. R. Pederson, Phys. Rev. B 54, 7830 (1996).

[19] U. J. Kim, C. A. Furtado, X. Liu, G. Chen, and P. C. Eklund, J. Am. Chem. Soc. 127, 15437 (2005).

[20] U. J. Kim, X. M. Liu, C. A. Furtado, G. Chen, R. Saito, J. Jiang, M.S. Dresselhaus, and P. C. Eklund, Phys. Rev. Lett. 95, 157402 (2005).

[21] J. Zhou and J. Dong, J. Appl. Phys. 107, 024306 (2010).

[22] G. Luo, H. Li, L. Wang, L. Lai, J. Zhou, R. Qin, J. Lu, W.-N. Mei, and Z. Gao, J. Phys. Chem. C 114, 6959 (2010).

[23] The unit $\mathrm{km} / \mathrm{mol}$ refers to the integrated IR intensity. Hence, to obtain the values from the plots in Ref. [22], the peaks must be integrated, or, equivalently, have their maxima multiplied by $2 / \pi \gamma$, where $\gamma=20 \mathrm{~cm}^{-1}$.

[24] W. Plank, R. Pfeiffer, C. Schaman, H. Kuzmany, M. Calvaresi, F. Zerbetto, and J. Meyer, ACS Nano 4, 4515 (2010). 